\title{
MIC Distributions and Evaluation of Fungicidal Activity for Amphotericin B, Itraconazole, Voriconazole, Posaconazole and Caspofungin and 20 Species of Pathogenic Filamentous Fungi Determined Using the CLSI Broth Microdilution Method
}

\author{
Andrew M. Borman *, Mark Fraser, Michael D. Palmer, Adrien Szekely, Marian Houldsworth, \\ Zoe Patterson and Elizabeth M. Johnson \\ Public Health England United Kingdom, Mycology Reference Laboratory, Myrtle Road, Bristol BS2 8EL, UK; \\ mark.fraser@phe.gov.uk (M.F.); michael.palmer@phe.gov.uk (M.D.P); adrien.szekely@phe.gov.uk (A.S.); \\ marian.houldsworth@phe.gov.uk (M.H.); zoe.patterson@phe.gov.uk (Z.P.); \\ elizabeth.johnson@phe.gov.uk (E.M.J.) \\ * Correspondence: andrew.borman@phe.gov.uk; Tel.: +44-114-3425032
}

Academic Editors: Ana Alastruey-Izquierdo and Stéphane Ranque

Received: 2 May 2017; Accepted: 24 May 2017; Published: 31 May 2017

\begin{abstract}
For filamentous fungi (moulds), species-specific interpretive breakpoints and epidemiological cut-off values (ECVs) have only been proposed for a limited number of fungal species-antifungal agent combinations, with the result that clinical breakpoints are lacking for most emerging mould pathogens. In the current study, we have compiled minimum inhibitory concentration (MIC) data for 4869 clinical mould isolates and present full MIC distributions for amphotericin B, itraconazole, voriconazole, posaconazole, and caspofungin with these isolates which comprise 20 species/genera. In addition, we present the results of an assessment of the fungicidal activity of these same five antifungal agents against a panel of 123 mould isolates comprising 16 of the same species.
\end{abstract}

Keywords: filamentous fungi; susceptibility testing; ECVs; amphotericin B; itraconazole; posaconazole; voriconazole; caspofungin; fungicidal; wild-type distributions

\section{Introduction}

Infections caused by filamentous fungi (moulds) are increasingly common in immunocompromised hosts, and are associated with high morbidity and mortality [1]. Although Aspergillus fumigatus and other Aspergillus species remain the most common causal agents [2-4], disseminated infections caused by Fusarium spp., Scedosporium spp., and members of the Mucoromycotina (Rhizopus, Lichtheimia, Mucor spp.) and other rarer moulds are associated with particularly poor prognoses [5-13]. The emergence of new fungal pathogens has been mirrored by an increasing number of species that are resistant to at least some of the established antifungal agents [14-17]. As a result of these trends, new antifungal agents continue to find significant clinical use, and the spectrum of antifungal agents continues to grow [18,19].

Given the range of fungal pathogens and the number of available antifungal agents, in vitro antifungal susceptibility testing of individual isolates is recommended for invasive fungal infections in high-risk patients, both in order to optimise treatment strategies and to detect resistant isolates. However, despite the existence of standardised broth microdilution methodologies for the susceptibility testing of filamentous fungi [20,21], species-specific interpretive breakpoints and epidemiological cut-off values (ECVs) have only been proposed for a limited number of fungal species-antifungal agent combinations [22-27]. 
Mainly on the basis of in vitro testing, the activity of the various antifungal agents has been described as fungistatic or fungicidal, depending on the drug, isolate, and test conditions. Amphotericin $B$ has been the most widely used antifungal agent against systemic infections, and exhibits concentration-dependent fungicidal activity against most fungal isolates in vitro [28]. Similarly, the echinocandins inhibit $\beta$-1,3-D-glucan synthesis, and are fungicidal against many Candida strains [29], but fungistatic against Aspergillus spp. Conversely, the azoles-itraconazole and voriconazole-have been described as exhibiting concentration-dependent fungistatic activity against many organisms [28] and fungicidal activity against a selection of others [30-32]. At least certain of these results have been confirmed in experimental models of infection in neutropenic rabbits, guinea pigs, and mice (reviewed in [32]).

Today, much uncertainty remains as to the best in vitro indicator of clinical outcome or in vivo susceptibility. For filamentous fungi, with the exception of the minimum inhibitory concentrations (MICs) for Aspergillus spp. with the azoles [33], few correlates between MIC and in vivo outcome have been described [34]. A relationship between voriconazole MIC and in vivo efficacy was noted in immunocompromised mice [35], and reasonable in vitro-in vivo correlates do exist for the fluconazole, caspofungin, and amphotericin B minimum fungicidal concentrations (MFCs) obtained with Candida spp. and Aspergillus spp., at least in animal models of invasive fungal disease (reviewed in [32]). A number of previous studies have attempted to examine the in vitro relationships between MIC and MFC for a limited number of fungal species, each with a subset of antifungal agents, in some cases employing non-standardised methodologies [31,36-38]. However, for filamentous fungi, a clear beneficial effect of treatment with fungicidal agents over fungistatic ones on clinical outcome remains unproven $[39,40]$.

Here, we present the MIC distributions for amphotericin B, itraconazole, voriconazole, caspofungin, and posaconazole for 20 species of filamentous fungi (4869 isolates) that were submitted to the UK Mycology Reference Laboratory (MRL) for susceptibility testing by the CLSI broth microdilution method. In addition, we have evaluated the fungicidal activities of those same five antifungals against a subset of 123 fungal isolates representing 16 of these species.

\section{Materials and Methods}

\subsection{Clinical Isolates for Minimum Inhibitory/Effective Concentration (MIC/MEC) Distribution Analyses}

Minimum inhibitory/effective concentration (MIC/MEC) distributions were ascertained for 4869 clinical isolates of filamentous fungi submitted to the MRL for susceptibility testing between 2006-2016. Isolates included Acremonium spp. ( $n=55)$, Alternaria spp. (58), Aspergillus fumigatus (2501), Aspergillus flavus (372), Aspergillus niger (301), Aspergillus terreus (115), Aspergillus versicolor (28), Exophiala spp. (134), Fusarium spp. (586), Lichtheimia corymbifera (64), Lomentospora prolificans (25), Mucor spp. (80), Paecilomyces variotii (33), Purpureocillium lilacinum (42), Rasamsonia spp. (29), Rhizopus arrhizus (21), Rhizopus microsporus (50), Rhizomucor pusillus (34), and Scedosporium apiospermum species complex (301). Isolates were identified phenotypically according to standard protocols in our laboratory. MICs and MECs were determined according to CLSI guidelines [20] by broth microdilution as described below.

\subsection{Additional Isolates}

For determination of fungicidal activities, the vast majority of mould isolates came from the National Collection of Pathogenic Fungi (NCPF) housed at the MRL, Bristol, although four strains of Aspergillus terreus and three strains of Aspergillus niger were recent clinical isolates. For moulds, two reference isolates of $A$. fumigatus (NCPF7097 and NCPF7100) were included in all assays as quality controls. The moulds tested comprised 11 isolates of Aspergillus fumigatus, 10 isolates each of Scedosporium apiospermum, Lomentospora prolificans, Aspergillus flavus, Aspergillus terreus, Lichtheimia corymbifera, 9 isolates each of Aspergillus niger, Exophiala dermatitidis, and Fusarium solani, 13 isolates of Fusarium spp. (6 isolates of F. proliferatum, 4 of F. oxysporum, and 3 of F. verticilloides), 7 isolates 
each of Purpureocillium lilacinum and Paecilomyces variotii, 4 isolates of Rhizopus arrhizus, 3 isolates of Rhizomucor pusillus, and 1 isolate of Rhizopus microsporus. NCPF isolates were retrieved from storage in liquid nitrogen or water, subcultured on plates of Oxoid Sabouraud dextrose agar (Unipath Ltd., Basingstoke, UK) supplemented with $0.5 \%(w / v)$ chloramphenicol, and incubated at $30^{\circ} \mathrm{C}$. Mould strains were subcultured onto slopes of Oxoid potato dextrose agar and incubated at $35^{\circ} \mathrm{C}$ for 7 days to induce sporulation, prior to testing.

\subsection{Antifungal Agents and Drug Concentration Ranges}

Antifungal drugs were obtained from their respective manufacturers as standard powders. Amphotericin B (Sigma Chemical Co., St. Louis, MO, USA) and voriconazole (Pfizer Central Research, Sandwich, UK) were dissolved in dimethyl sulfoxide. Itraconazole (Janssen Research Foundation, Beerse, Belgium) and posaconazole (Merck, Sharp and Dohme, Hoddesdon, UK) were dissolved in PEG400 by heating to $70^{\circ} \mathrm{C}$. Caspofungin (Merck, Sharp and Dohme) was resuspended in sterile water. Serial two-fold dilutions of the various drugs were prepared in RPMI 1640 medium (with L-glutamine, without bicarbonate; Sigma Chemical Co.) and buffered to $\mathrm{pH} 7.0$ using a $0.165 \mathrm{M}$ solution of MOPS (Sigma Chemical Co.). The antifungal agents were tested over a range of final concentrations $(0.03$ to $16 \mu \mathrm{g} / \mathrm{mL}$ for amphotericin B, voriconazole, posaconazole, and itraconazole; 0.125 to $64 \mu \mathrm{g} / \mathrm{mL}$ for caspofungin).

\subsection{CLSI Broth Microdilution Determination of Mould MIC and MEC}

MICs were determined in round-bottomed 96-well plates with mould conidial suspensions prepared in RPMI 1640 and adjusted to a final concentration of $(0.4-5) \times 10^{4} \mathrm{CFU} / \mathrm{mL}$ as previously described [20]. Inoculated plates were incubated for $48 \mathrm{~h}$ at $35^{\circ} \mathrm{C}$ except for L. corymbifera, R. arrhizus, R. pusillus, and R. microsporus, which were incubated for $24 \mathrm{~h}$. MICs were read at 24 or $48 \mathrm{~h}$ as the concentration of drug that elicited 100\% inhibition of growth (amphotericin B, itraconazole, posaconazole, voriconazole) or as the minimum effective concentration (MEC, caspofungin), in which the end-point is read as the lowest concentration at which the fungal hyphae can be seen to be stunted with swollen tips.

\subsection{Determination of Minimum Fungicidal Concentrations}

MFCs were determined after $48 \mathrm{~h}$ incubation (except for L. corymbifera, R. arrhizus, R. pusillus, and R. microsporus, which were determined at $24 \mathrm{~h}$ ) by removing $10 \mu \mathrm{L}$ of the contents from wells showing no visible growth and spreading them on to Sabouraud dextrose agar plates. The plates were then incubated for $72 \mathrm{~h}$ and MFCs were determined as the lowest drug concentrations which killed $95 \%$ of the inoculum.

\subsection{Data Analysis}

MIC and MFC ranges and the drug concentrations required to inhibit or kill 50\% (MIC 50 and $\mathrm{MFC}_{50}$, respectively) or $90 \%$ of isolates ( $\mathrm{MIC}_{90}$ and $\mathrm{MFC}_{90}$, respectively) were determined for all species that comprised at least seven isolates. For species comprising less than seven isolates, only MIC and MFC ranges were determined.

\section{Results}

The results of in vitro susceptibility testing of 4689 clinical isolates of filamentous fungi submitted to the MRL are summarised in Tables 1-5. In all tests included in the data analysis, the MICs of the control reference strains were within the accepted limits (data not shown). CLSI wild-type MIC distributions and ECVs have only been proposed for Aspergillus spp. [23,25-27], and some members of the Mucoromycotina [22]. For the more commonly encountered Fusarium spp., ECVs have been proposed using CLSI methodology [41], but have not been formally accepted by CLSI. In the current 
study, we could not establish wild-type distributions or ECVs for the various organisms studied, as under CLSI guidelines they can only be defined when more than 100 MIC results per species are available from at least three independent laboratories.

Table 1. Minimum inhibitory concentration (MIC) distributions for amphotericin B and common pathogenic filamentous fungi. The number of isolates included is given in parentheses, together with the number of isolates with each given MIC value. \%R denotes the proportion of isolates with non-wild type MICs (i.e., MICs greater than the CLSI 97.5\% epidemiological cutoff value (ECV) proposed for $A$. fumigatus, dashed line [23],) with percentages above 30\% in bold face. Dashed Line: no isolates with this MIC.

\begin{tabular}{|c|c|c|c|c|c|c|c|c|c|c|c|}
\hline \multirow{2}{*}{ Antifungal/Organism } & \multicolumn{11}{|c|}{ MIC (mg/L) } \\
\hline & 0.03 & 0.06 & 0.125 & 0.25 & 0.5 & 1 & 2 & 4 & 8 & 16 & $\% \mathrm{R}$ \\
\hline \multicolumn{12}{|l|}{ Amphotericin B } \\
\hline Acremonium sp. (55) & - & 1 & - & 2 & 5 & 9 & 15 & 15 & 3 & 5 & 69.1 \\
\hline Alternaria sp. (58) & - & 3 & 7 & 24 & 20 & 4 & - & - & - & - & 0 \\
\hline Aspergillus flavus (372) & - & - & 2 & 16 & 104 & 199 & 42 & 9 & - & - & 13.7 \\
\hline Aspergillus fumigatus (2501) & - & 1 & 11 & 134 & 778 & 1265 & 310 & 2 & - & - & 12.5 \\
\hline Aspergillus nidulans (40) & 2 & 1 & 2 & 8 & 13 & 12 & 2 & - & - & - & 5 \\
\hline Aspergillus niger (301) & 6 & 49 & 117 & 101 & 27 & 1 & - & - & - & - & 0 \\
\hline Aspergillus terreus (115) & - & - & - & 2 & 16 & 42 & 43 & 7 & 5 & - & 47.8 \\
\hline Aspergillus versicolor (28) & - & - & - & 2 & 9 & 10 & 7 & - & - & - & 25 \\
\hline Exophiala sp. (134) & 1 & 2 & 14 & 33 & 67 & 17 & - & - & - & - & 0 \\
\hline Fusarium sp. (586) & - & 1 & 3 & 6 & 71 & 304 & 153 & 40 & 8 & - & 34.3 \\
\hline Lichtheimia corymbifera (64) & 1 & 5 & 13 & 28 & 13 & 4 & - & - & - & - & 0 \\
\hline Lomentospora prolificans (25) & - & - & - & - & - & - & 5 & 8 & 5 & 7 & 100 \\
\hline Mucor sp. (80) & 5 & 9 & 22 & 21 & 19 & 3 & - & - & 1 & - & 1.2 \\
\hline Paecilomyces variotii (33) & 3 & 10 & 5 & 6 & 3 & 4 & 2 & - & - & - & 6.1 \\
\hline Purpureocillium lilacinum (42) & - & - & - & - & - & - & 1 & - & 1 & 40 & 100 \\
\hline Rasamsonia sp. (29) & - & - & 1 & - & 5 & 8 & 6 & 6 & 3 & - & 31 \\
\hline Rhizopus arrhizus (21) & - & 1 & 5 & 6 & 7 & 2 & - & - & - & - & 0 \\
\hline Rhizopus microsporus (50) & - & 1 & 6 & 19 & 20 & 3 & 1 & - & - & - & 2 \\
\hline Rhizomucor pusillus (34) & 1 & 1 & 7 & 18 & 7 & - & - & - & - & - & 0 \\
\hline Scedosporium apiospermum (301) & - & 1 & 3 & 10 & 34 & 94 & 65 & 58 & 14 & 22 & 52.8 \\
\hline
\end{tabular}

MIC data for amphotericin B were broadly concordant with previously published international studies $[22,23,41]$. Organisms for which significant proportions of isolates had MICs in excess of the ECV for A. fumigatus included A. terreus and Fusarium spp., for which higher ECVs have been proposed [23,41] and Lomentospora prolificans, Purpureocillium lilacinum, Rasamsonia spp. and Scedosporium apiospermum species complex. Previous studies have highlighted elevated amphotericin B MICs with these organisms [42-44], and amphotericin B is not currently suggested as optimal first line treatment for those organisms [45]. For the Mucoromycotina, MIC ranges were predominantly below the ECV of A. fumigatus, in agreement with previous studies that specifically aimed to define ECVs for these organisms [22].

With itraconazole, again most isolates of many of the species examined had MICs below the ECV proposed for A. fumigatus (Table 2). Organisms with heavily skewed MIC distributions included Acremonium spp., Fusarium spp., and Lo. prolificans. Once again, current treatment guidelines do not recommend itraconazole for infections by these organisms [45]. In agreement with previous studies involving the Mucoromycotina [22], the MICs for Mucor spp., Rhizopus spp., and Rhizomucor pusillus were considerably higher than those observed with L. corymbifera, with concomitantly higher ECVs having been proposed for the former organisms [22]. It should be noted that approximately $5 \%$ of isolates of A. fumigatus from the current study had MICs above the proposed ECV of $1 \mathrm{mg} / \mathrm{L}$ with itraconazole. This is likely to result from patient bias in the samples received by the MRL, where a high proportion of isolates are from patients with cystic fibrosis and are thus likely to have had previous itraconazole exposure (data not shown). 
Table 2. MIC distributions for Iitraconazole. For presentation conventions, see Legend to Table 1.

\begin{tabular}{|c|c|c|c|c|c|c|c|c|c|c|c|c|}
\hline \multirow{2}{*}{ Antifungal/Organism } & \multicolumn{12}{|c|}{ MIC (mg/L) } \\
\hline & 0.03 & 0.06 & 0.125 & 0.25 & 0.5 & 1 & 2 & 4 & 8 & 16 & 32 & $\% R$ \\
\hline \multicolumn{13}{|l|}{ Itraconazole } \\
\hline Acremonium sp. (50) & - & 1 & 1 & 3 & 3 & 7 & 2 & 3 & 3 & - & 27 & 70 \\
\hline Alternaria sp. (68) & 3 & 7 & 20 & 28 & 8 & 1 & 1 & - & - & - & - & 1.5 \\
\hline Aspergillus flavus (244) & 34 & 91 & 82 & 29 & 8 & - & - & - & - & - & - & 0 \\
\hline Aspergillus fumigatus (2268) & 22 & 453 & 629 & 632 & 370 & 53 & 24 & 21 & 9 & 45 & 10 & 4.8 \\
\hline Aspergillus nidulans (39) & 7 & 9 & 14 & 9 & - & - & - & - & - & - & - & 0 \\
\hline Aspergillus niger (149) & 1 & 6 & 40 & 48 & 51 & 10 & 3 & - & - & - & - & 2 \\
\hline Aspergillus terreus (98) & 23 & 41 & 25 & 5 & 4 & - & - & - & - & - & - & 0 \\
\hline Aspergillus versicolor (21) & 2 & 1 & 4 & 6 & 6 & 1 & 1 & - & - & - & - & 4.7 \\
\hline Exophiala sp. (139) & 7 & 12 & 34 & 51 & 26 & 7 & 2 & - & - & - & - & 1.4 \\
\hline Fusarium sp. (488) & - & - & - & 4 & 18 & 16 & 26 & 18 & 2 & 404 & - & 98.2 \\
\hline Lichtheimia corymbifera (56) & 2 & 2 & 13 & 24 & 14 & 1 & - & - & - & - & - & 0 \\
\hline Lomentospora prolificans (25) & - & - & - & - & - & - & - & - & - & 25 & - & 100 \\
\hline Mucor sp. (76) & 2 & 2 & 1 & 4 & 12 & 15 & 7 & 1 & - & 32 & - & 52.6 \\
\hline Paecilomyces variotii (30) & 11 & 7 & 2 & 3 & 1 & 1 & 2 & 1 & 2 & - & - & 16.7 \\
\hline Purpureocillium lilacinum (38) & 1 & - & - & 3 & 20 & 11 & - & 2 & - & 2 & - & 10.5 \\
\hline Rasamsonia sp. (30) & 1 & - & 2 & 2 & 10 & 8 & 4 & 2 & - & 1 & - & 23.3 \\
\hline Rhizopus arrhizus (19) & - & - & - & 1 & 4 & 5 & 3 & 1 & 1 & 4 & - & 47.4 \\
\hline Rhizopus microsporus (46) & - & - & - & 4 & 16 & 15 & 7 & - & - & 4 & - & 23.9 \\
\hline Rhizomucor pusillus (29) & 2 & 4 & - & 7 & 7 & 3 & 3 & - & - & 3 & - & 20.7 \\
\hline Scedosporium apiospermum (287) & 3 & 7 & 23 & 89 & 111 & 29 & 7 & 8 & 2 & 8 & - & 8.7 \\
\hline
\end{tabular}

Posaconazole MIC distributions are given in Table 3. MIC ranges significantly higher than the ECV for A. fumigatus were observed with Fusarium spp., Lo. prolificans, and certain members of the Mucoromycotina, in broad agreement with previous reports $[8,22,41]$. Of the antifungal agents studied here, amphotericin B and posaconazole appear to exert the most activity against the Mucoromycotina in vitro, in agreement with previous studies and current treatment recommendations [8].

Table 3. MIC distributions for posaconazole. For presentation conventions, see Legend to Table 1.

\begin{tabular}{lcccccccccccc}
\hline \multirow{2}{*}{ Antifungal/organism } & \multicolumn{1}{c}{ MIC (mg/L) } \\
\cline { 2 - 11 } & $\mathbf{0 . 0 3}$ & $\mathbf{0 . 0 6}$ & $\mathbf{0 . 1 2 5}$ & $\mathbf{0 . 2 5}$ & $\mathbf{0 . 5}$ & $\mathbf{1}$ & $\mathbf{2}$ & $\mathbf{4}$ & $\mathbf{8}$ & $\mathbf{1 6}$ & $\mathbf{\%}$ \\
\hline Posaconazole & & & & & & & & & & \\
Acremonium sp. (8) & - & 1 & 2 & 1 & 1 & 3 & - & - & - & - & 37.5 \\
Alternaria sp. (32) & 7 & 18 & 6 & 1 & - & - & - & - & - & - & 0 \\
Aspergillus flavus (83) & 21 & 44 & 15 & 3 & - & - & - & - & - & - & 0 \\
Aspergillus fumigatus (396) & 111 & 150 & 65 & 37 & 21 & 8 & 2 & - & 1 & 1 & 3 \\
Aspergillus nidulans (7) & 1 & 4 & 1 & 1 & - & - & - & - & - & - & 0 \\
Aspergillus niger (43) & 3 & 25 & 10 & 4 & 1 & - & - & - & - & - & 0 \\
Aspergillus terreus (37) & 18 & 15 & 3 & - & - & - & 1 & - & - & - & 2.7 \\
Aspergillus versicolor (7) & 1 & 1 & 1 & 2 & 2 & - & - & - & - & - & 0 \\
Exophiala sp. (34) & 3 & 11 & 12 & 4 & 4 & - & - & - & - & - & 0 \\
Fusarium sp. (124) & - & - & 3 & 6 & 19 & 19 & 18 & 5 & 1 & 53 & 77.4 \\
Lichtheimia corymbifera (58) & - & 3 & 16 & 26 & 13 & - & - & - & - & - & 0 \\
Lomentospora prolificans (21) & - & - & - & - & 1 & 1 & 3 & - & 16 & - & $\mathbf{9 5 . 2}$ \\
Mucor sp. (79) & 3 & 3 & 3 & 9 & 18 & 21 & 10 & 6 & 1 & 5 & $\mathbf{4 1 . 8}$ \\
Paecilomyces variotii (19) & 9 & 5 & 1 & 1 & 2 & - & 1 & - & - & - & 5.5 \\
Purpureocillium lilacinum (13) & 1 & 4 & 2 & 5 & - & 1 & - & - & - & - & 7.7 \\
Rasamsonia sp. (13) & 1 & - & 2 & - & 8 & 2 & - & - & - & - & 15.4 \\
Rhizopus arrhizus (20) & - & - & 2 & 3 & 6 & 7 & - & - & - & 2 & $\mathbf{4 5}$ \\
Rhizopus microsporus (49) & - & 1 & 1 & 13 & 27 & 1 & 3 & 2 & - & 1 & 14.3 \\
Rhizomucor pusillus (32) & - & 7 & 2 & 5 & 12 & 4 & 1 & 1 & - & - & 18.7 \\
Scedosporium apiospermum (85) & 2 & 2 & 10 & 39 & 26 & 2 & 1 & 1 & - & 2 & 7 \\
\hline
\end{tabular}

Depending on infection site, voriconazole is recommended as first-line treatment for infections with S. apiospermum, Aspergillus spp., Acremonium spp., Fusarium spp., and Lo. prolificans (in combination 
with terbinafine) [45]. For Acremonium spp., S. apiospermum, and all of the Aspergillus spp. examined here, MIC values predominantly fell below the ECV proposed for A. fumigatus [26] (Table 4). For Fusarium spp., MICs were lower with voriconazole than with either itraconazole or posaconazole, and clinical treatment successes have been observed with voriconazole-especially when accompanied by surgical interventions and immune reconstitution [45]. Similarly, voriconazole is superior to itraconazole and posaconazole in vitro towards Lo. prolificans, and has been associated with $40-50 \%$ survival rates especially when used in conjunction with terbinafine [45]. Voriconazole had little apparent in vitro activity against the remaining species examined here (members of the Mucoromycotina, P. variotii, Rasamsonia spp.), with MIC ranges significantly higher than the ECV for A. fumigatus.

Table 4. MIC distributions for voriconazole. For presentation conventions, see Legend to Table 1.

\begin{tabular}{|c|c|c|c|c|c|c|c|c|c|c|c|}
\hline \multirow{2}{*}{ Antifungal/organism } & \multicolumn{11}{|c|}{ MIC (mg/L) } \\
\hline & 0.03 & 0.06 & 0.125 & 0.25 & 0.5 & 1 & 2 & 4 & 8 & 16 & $\% R$ \\
\hline \multicolumn{12}{|l|}{ Voriconazole } \\
\hline Acremonium sp. (55) & - & 2 & 5 & 11 & 20 & 11 & 3 & 1 & 1 & 1 & 10.9 \\
\hline Alternaria sp. (63) & - & - & 7 & 6 & 13 & 20 & 6 & 8 & 3 & 1 & 28.6 \\
\hline Aspergillus flavus (283) & - & 5 & 72 & 154 & 47 & 3 & 1 & 1 & - & - & 0.7 \\
\hline Aspergillus fumigatus (2384) & 1 & 13 & 309 & 1631 & 299 & 66 & 45 & 13 & - & 7 & 3.1 \\
\hline Aspergillus nidulans (39) & 2 & 14 & 19 & 2 & 2 & - & - & - & - & - & 0 \\
\hline Aspergillus niger (164) & 2 & 3 & 24 & 82 & 47 & 6 & - & - & - & - & 0 \\
\hline Aspergillus terreus (106) & - & 1 & 31 & 55 & 16 & 1 & 2 & - & - & - & 1.9 \\
\hline Aspergillus versicolor (22) & - & 3 & 7 & 5 & 6 & - & 1 & - & - & - & 4.5 \\
\hline Exophiala sp. (126) & 23 & 20 & 42 & 25 & 14 & 8 & 11 & 1 & 2 & - & 11.1 \\
\hline Fusarium sp. (593) & 2 & 2 & 11 & 11 & 46 & 151 & 185 & 153 & 31 & 1 & 62.4 \\
\hline Lichtheimia corymbifera (50) & - & - & - & - & - & - & 7 & 12 & 16 & 15 & 100 \\
\hline Lomentospora prolificans (25) & - & - & - & - & - & 9 & 10 & 6 & - & - & 64 \\
\hline Mucor sp. (73) & - & - & 2 & - & - & - & 1 & 3 & 4 & 63 & 97.3 \\
\hline Paecilomyces variotii (30) & - & - & - & 2 & 2 & 1 & 10 & 2 & 1 & 12 & 83.3 \\
\hline Purpureocillium lilacinum (43) & 2 & 2 & 22 & 13 & 3 & - & - & - & 1 & - & 2.3 \\
\hline Rasamsonia sp. (30) & - & - & - & - & - & - & - & 3 & - & 27 & 100 \\
\hline Rhizopus arrhizus (18) & - & - & - & - & - & - & 2 & 3 & 6 & 7 & 100 \\
\hline Rhizopus microsporus (36) & - & - & - & - & - & - & 1 & 15 & 17 & 2 & 100 \\
\hline Rhizomucor pusillus (30) & - & - & 1 & - & - & 3 & 1 & 3 & 5 & 17 & 86.7 \\
\hline Scedosporium apiospermum (304) & 9 & 51 & 101 & 114 & 19 & 1 & 7 & 1 & 1 & - & 2.9 \\
\hline
\end{tabular}

The MEC ranges observed with caspofungin were significantly higher than the proposed ECV for A. fumigatus for all organisms except Aspergillus spp. and Rasamsonia spp. (Table 5). For A. nidulans and to a lesser extent $A$. versicolor, MEC ranges appeared to be bimodal as described previously [23], with a small proportion of isolates having MECs significantly above the A. fumigatus ECV. The apparent lack of in vitro activity of caspofungin against Fusarium spp., members of the Mucoromycotina, $S$. apiospermum, Lo. prolificans, P. variotii, P. lilacinum, and Exophiala spp. has been reported previously [46], and correlates well with the lack of in vivo success of the echinocandin antifungal agents as primary treatment of infections with these various organisms, which presumably is a result of the reduced concentrations of $1,3-\beta$-D-glucan in the cell walls of many of these species $[47,48]$.

Certain studies have suggested that in vivo outcome may be improved when fungicidal as opposed to fungistatic agents are employed for treatment, although the evidence with invasive mould infections is less than compelling [39]. To evaluate potential species-specific differences in the fungicidal versus fungistatic actions of the five antifungal agents included in the current study, a subset of 123 isolates from the NCPF were subjected to susceptibility testing by CLSI broth microdilution followed by end-point plating to determine MFCs (see Section 2). The ranges of MICs for the various drug-organism combinations were indistinguishable from those observed in our analyses of the 4869 clinical isolates referred to the MRL from various UK hospital centre microbiology laboratories (data not shown). 
Table 5. Minimum effective concentration (MEC) distributions for caspofungin. For presentation conventions, see Legend to Table 1.

\begin{tabular}{|c|c|c|c|c|c|c|c|c|c|c|c|c|c|}
\hline \multirow{2}{*}{ Antifungal/Organism } & \multicolumn{13}{|c|}{$\operatorname{MEC~(mg/L)~}$} \\
\hline & 0.015 & 0.03 & 0.06 & 0.125 & 0.25 & 0.5 & 1 & 2 & 4 & 8 & 16 & 32 & $\% \mathbf{R}$ \\
\hline \multicolumn{14}{|l|}{ Caspofungin } \\
\hline Acremonium sp. (11) & - & - & - & - & 1 & 1 & 1 & 3 & 1 & - & 1 & 3 & 72.7 \\
\hline Alternaria sp. (20) & - & - & - & 1 & 5 & 5 & 3 & 1 & - & - & 1 & 3 & 25 \\
\hline Aspergillus flavus (134) & 15 & 12 & 6 & 14 & 74 & 5 & 3 & 4 & - & - & - & 1 & 3.7 \\
\hline Aspergillus fumigatus (946) & 2 & 64 & 100 & 86 & 594 & 89 & - & - & 1 & 2 & 4 & 4 & 1.2 \\
\hline Aspergillus nidulans (18) & - & - & 2 & 2 & 6 & 2 & 1 & 4 & 1 & - & - & - & 27.8 \\
\hline Aspergillus niger (83) & - & 13 & 7 & 9 & 48 & 6 & - & - & - & - & - & - & 0 \\
\hline Aspergillus terreus (43) & - & 2 & 5 & 4 & 32 & - & - & - & - & - & - & - & 0 \\
\hline Aspergillus versicolor (17) & - & 2 & 1 & 1 & 5 & 5 & - & 1 & 2 & - & - & - & 17.6 \\
\hline Exophiala sp. (33) & 1 & - & - & - & 1 & - & 1 & 7 & 2 & 4 & 1 & 16 & 90.9 \\
\hline Fusarium sp. (81) & - & - & - & - & 2 & 1 & - & 2 & 5 & 9 & 12 & 50 & 96.3 \\
\hline Lichtheimia corymbifera (10) & - & - & - & - & - & - & - & - & - & 1 & - & 9 & 100 \\
\hline Lomentospora prolificans (10) & - & - & - & - & - & - & - & - & 2 & 3 & 4 & 1 & 100 \\
\hline Mucor sp. (13) & - & - & - & - & - & - & - & - & - & - & - & 13 & 100 \\
\hline Paecilomyces variotii (16) & - & - & 1 & 1 & 4 & 3 & - & 5 & 1 & - & 1 & - & 43.7 \\
\hline Purpureocillium lilacinum (6) & - & - & - & 1 & - & 1 & - & 2 & 1 & - & - & 1 & 66.7 \\
\hline Rasamsonia sp. (5) & 1 & 1 & 1 & 2 & - & - & - & - & - & - & - & - & 0 \\
\hline Rhizopus arrhizus (7) & - & - & - & - & - & - & - & - & - & - & 2 & 5 & 100 \\
\hline Rhizopus microsporus (10) & - & - & - & - & - & - & - & - & - & 1 & - & 9 & 100 \\
\hline Rhizomucor pusillus (5) & - & - & - & - & - & - & - & - & - & - & - & 5 & 100 \\
\hline Scedosporium apiospermum (98) & - & - & 2 & 2 & 4 & 4 & 20 & 33 & 4 & 10 & 3 & 13 & 64.3 \\
\hline
\end{tabular}

The MFC ranges for the five different drugs were, however, significantly different for the various organisms (Table 6). Amphotericin B exhibited fungicidal activity against isolates of A. fumigatus, A. flavus, A. niger, E. dermatitidis, L. corymbifera, $R$. arrhizus, and $R$. microsporus $\left(\mathrm{MFC}_{50}\right.$ s where appropriate $<1 \mu \mathrm{g} / \mathrm{mL}$ ). Indeed, the MFCs of amphotericin B for these organisms rarely exceeded the modal MICs observed in our larger survey by more than 1-2 doubling dilutions (Table 6). Itraconazole, posaconazole, and voriconazole also exhibited significant fungicidal activity, killing at least $50 \%$ of isolates of A. fumigatus, A. flavus, A. niger, A. terreus, and E. dermatitidis. Voriconazole and posaconazole were the only antifungal drugs tested to demonstrate any fungicidal activity against isolates of $S$. apiospermum or P. lilacinum, whereas the same was true for itraconazole and posaconazole with isolates of $P$. variotii. Posaconazole was the only triazole antifungal agent tested to exhibit at least limited fungicidal activity against some members of the Mucoromycotina. No significant fungicidal activity could be detected for any mould-drug combination with caspofungin.

Table 6. Minimum fungicidal concentration (MFC) data for mould isolates. Fungicidal activities are highlighted in grey (i.e., fungicidal effect observed at or below ECV MIC for A. fumigatus with that antifungal agent). na-Not appropriate due to the small number of isolates tested for the species. nd-Not done. * Fusarium spp. included F. proliferatum (6), F. verticillioides (3), and F. oxysporum (4) species complexes.

\begin{tabular}{ccccc}
\hline \multirow{2}{*}{ Species (No. Tested) } & Antifungal Agent & \multicolumn{3}{c}{ MFC $(\mathbf{m g} / \mathbf{m L})$} \\
\cline { 3 - 5 } & & Range & MFC $_{\mathbf{5 0}}$ & MFC $_{\mathbf{9 0}}$ \\
\hline S. apiospermum & Amphotericin B & $0.6->16$ & 2 & $>16$ \\
$(10)$ & Itraconazole & $0.5->16$ & 16 & $>16$ \\
& Voriconazole & $0.25->16$ & 1 & $>16$ \\
& Caspofungin & $4->64$ & 32 & $>64$ \\
& Posaconazole & $<0.03-0.5$ & 0.5 & 0.5 \\
\hline Lo. prolificans & Amphotericin B & $4->16$ & 8 & $>16$ \\
$(10)$ & Itraconazole & $>16$ & $>16$ & $>16$ \\
& Voriconazole & $>16$ & $>16$ & $>16$ \\
& Caspofungin & $16->64$ & 32 & $>64$ \\
& Posaconazole & $>16$ & $>16$ & $>16$ \\
\hline
\end{tabular}


Table 6. Cont

\begin{tabular}{|c|c|c|c|c|}
\hline \multirow{2}{*}{ Species (No. Tested) } & \multirow{2}{*}{ Antifungal Agent } & \multicolumn{3}{|c|}{ MFC (mg/mL) } \\
\hline & & Range & $\mathrm{MFC}_{50}$ & $\mathrm{MFC}_{90}$ \\
\hline \multirow{5}{*}{$\begin{array}{l}\text { F. solani } \\
\text { (9) }\end{array}$} & Amphotericin B & $0.25->16$ & 2 & 4 \\
\hline & Itraconazole & $>16$ & $>16$ & $>16$ \\
\hline & Voriconazole & $8->16$ & 16 & $>16$ \\
\hline & Caspofungin & $64->64$ & $>64$ & $>64$ \\
\hline & Posaconazole & $1->16$ & $>16$ & $>16$ \\
\hline \multirow{5}{*}{$\begin{array}{l}\text { Fusarium spp. * } \\
\text { (13) }\end{array}$} & Amphotericin B & $1-4$ & 2 & 4 \\
\hline & Itraconazole & $>16$ & $>16$ & $>16$ \\
\hline & Voriconazole & $4->16$ & 16 & 16 \\
\hline & Caspofungin & $>64$ & $>64$ & $>64$ \\
\hline & Posaconazole & $1->16$ & 2 & $>16$ \\
\hline \multirow{5}{*}{$\begin{array}{l}\text { A. fumigatus } \\
\text { (11) }\end{array}$} & Amphotericin B & $0.5-2$ & 1 & 2 \\
\hline & Itraconazole & $0.25->16$ & 0.5 & 2 \\
\hline & Voriconazole & $0.25-8$ & 1 & 4 \\
\hline & Caspofungin & $64->64$ & $>64$ & $>64$ \\
\hline & Posaconazole & $<0.03-0.25$ & $<0.03$ & 0.125 \\
\hline \multirow{5}{*}{$\begin{array}{l}\text { A. flavus } \\
\text { (10) }\end{array}$} & Amphotericin B & $1-2$ & 1 & 2 \\
\hline & Itraconazole & $0.06-0.25$ & 0.125 & 0.25 \\
\hline & Voriconazole & $0.25-8$ & 0.5 & 1 \\
\hline & Caspofungin & $8->64$ & 64 & $>64$ \\
\hline & Posaconazole & 0.06 & 0.06 & 0.06 \\
\hline \multirow{5}{*}{$\begin{array}{l}\text { A. niger } \\
\text { (9) }\end{array}$} & Amphotericin B & $0.25-2$ & 0.5 & 2 \\
\hline & Itraconazole & $0.25-0.5$ & 0.25 & 0.5 \\
\hline & Voriconazole & $0.25-1$ & 0.5 & 1 \\
\hline & Caspofungin & $32-64$ & 32 & 64 \\
\hline & Posaconazole & $<0.03-0.125$ & 0.06 & 0.125 \\
\hline \multirow{5}{*}{$\begin{array}{l}\text { A. terreus } \\
\text { (10) }\end{array}$} & Amphotericin B & $8->16$ & $>16$ & $>16$ \\
\hline & Itraconazole & $0.25-4$ & 0.5 & 4 \\
\hline & Voriconazole & $0.5->16$ & 2 & $>16$ \\
\hline & Caspofungin & $32->64$ & $>64$ & $>64$ \\
\hline & Posaconazole & $<0.03-1$ & 0.125 & 0.5 \\
\hline \multirow{5}{*}{$\begin{array}{l}\text { E. dermatitidis } \\
\text { (9) }\end{array}$} & Amphotericin B & $0.5-2$ & 0.5 & 2 \\
\hline & Itraconazole & $0.125-0.5$ & 0.25 & 0.5 \\
\hline & Voriconazole & $0.25->16$ & 0.5 & $>16$ \\
\hline & Caspofungin & $8->64$ & 32 & $>64$ \\
\hline & Posaconazole & nd & & \\
\hline \multirow{5}{*}{$\begin{array}{l}\text { P. variotii } \\
\text { (7) }\end{array}$} & Amphotericin B & $1->16$ & 16 & $>16$ \\
\hline & Itraconazole & $0.25-16$ & 0.5 & 16 \\
\hline & Voriconazole & $4->16$ & $>16$ & $>16$ \\
\hline & Caspofungin & $16-64$ & 16 & 64 \\
\hline & Posaconazole & $0.125-16$ & 0.25 & 16 \\
\hline \multirow{5}{*}{$\begin{array}{l}\text { P. lilacinum } \\
\text { (7) }\end{array}$} & Amphotericin B & $4->16$ & 8 & $>16$ \\
\hline & Itraconazole & $0.5->16$ & $>16$ & $>16$ \\
\hline & Voriconazole & $0.25-2$ & 1 & 2 \\
\hline & Caspofungin & $>64$ & $>64$ & $>64$ \\
\hline & Posaconazole (4) & $0.06-0.5$ & na & na \\
\hline \multirow{5}{*}{$\begin{array}{l}\text { L. corymbifera } \\
\text { (10) }\end{array}$} & Amphotericin B & $0.5->16$ & 1 & 4 \\
\hline & Itraconazole & $1->16$ & 4 & $>16$ \\
\hline & Voriconazole & $>16$ & $>16$ & $>16$ \\
\hline & Caspofungin & $>64$ & $>64$ & $>64$ \\
\hline & Posaconazole & $0.06->16$ & 0.25 & $>16$ \\
\hline
\end{tabular}


Table 6. Cont.

\begin{tabular}{ccccc}
\hline \multirow{2}{*}{ Species (No. Tested) } & Antifungal Agent & \multicolumn{3}{c}{ MFC (mg/mL) } \\
\cline { 3 - 5 } & & Range & MFC $_{\mathbf{5 0}}$ & MFC $_{\mathbf{9 0}}$ \\
\hline Rhizopus spp. & Amphotericin B & $0.25-1$ & na & na \\
(5) & Itraconazole & $0.25->16$ & na & na \\
& Voriconazole & $8->16$ & na & na \\
& Caspofungin & $>64$ & $>64$ & $>64$ \\
& Posaconazole & $0.125>16$ & na & na \\
\hline R.pusillus & Amphotericin B & $0.5-16$ & na & na \\
(3) & Itraconazole & $1->16$ & na & na \\
& Voriconazole & $>16$ & na & na \\
& Caspofungin & $>64$ & $>64$ & $>64$ \\
& Posaconazole & $>16$ & $>16$ & $>16$ \\
\hline
\end{tabular}

\section{Discussion}

The aim of antifungal susceptibility testing is to obtain a result than can be used to determine the likelihood of treatment success of an infection by a particular organism with the antifungal agent in question. Ideally, this interpretive decision relies upon the development of clinical breakpoints (CBPs) that are intended to predict therapeutic outcome. With yeast isolates, there is a suggestion that a "90:60 rule" should be applied, which proposes that susceptible organisms will respond to therapy $90 \%$ of the time whilst resistant organisms would only respond in $60 \%$ of cases [49]. As most patients with invasive mould infection are immunocompromised, the lack of adjunctive host response is likely to have an additional impact on the probable outcome of therapy. Indeed, in most clinical trials of invasive aspergillosis, there appears to be an irreducible $40 \%$ mortality rate even with susceptible organisms [50], and there is a wealth of anecdotal evidence and case histories to support the contention that mould isolates that are resistant in vitro will rarely-if ever-respond to therapy. Therefore, for moulds, perhaps the rule needs revision to a "10:60 rule" in which susceptible organisms will respond in $60 \%$ of cases and resistant organisms will only respond in $10 \%$.

Unfortunately, the development of CBPs is currently impossible for many filamentous fungal (mould) infections, principally due to the lack of sufficient clinical trial data for some of the rarer, emerging pathogens. In the absence of CBPs, ECVs can be used to determine whether a particular isolate may have acquired resistance to a particular antifungal agent via the identification of isolates with "non-wild-type" MICs. The current study was not geared towards developing ECVs, since they require the analysis of at least 100 independent isolates of a particular species, with MIC values obtained from at least three independent centres. However, the current study is intended to contribute to the existing literature and aid the future development of ECVs and CBPs via the analysis of the antifungal susceptibility profiles of a large number of clinical isolates from across the UK. Moreover, in the absence of specific antifungal susceptibility testing on the causative organism, the current data may help clinicians dealing with infections with rarer moulds to decide which antifungal drug may be most likely to be effective. The MIC distributions reported here for Aspergillus spp., Fusarium spp., and members of the Mucoromycotina are largely similar to those previously reported from large international studies involving the USA [23,25-27,41], suggesting that UK isolates are broadly similar to their US counterparts. Additional studies have suggested species-specific antifungal profiles and individual ECVs for different Fusarium spp. and for individual cryptic species within the S. apiospermum species complex [41,42]. Unfortunately, it was beyond the remit of the current study to identify individual Fusarium and S. apiospermum species to cryptic species level, and MIC data were grouped for the whole series in each case. Nevertheless, the MIC distributions reported here are very similar to those reported previously with individual species [41,42].

In the absence of ECV and CBP data, MIC distributions may still be clinically useful in identifying bimodal distributions indicative of sub-populations with acquired or intrinsic resistance. Similarly, identification of antifungal agent-organism combinations where MIC ranges are always elevated 
compared to other species may aid in eliminating particular therapeutic approaches. A number of such combinations including both yeast and mould species have become evident over the last decades, and will not be discussed in detail here. Examples include members of the Mucoromycotina, Scedosporium spp., Lo. prolificans, and Fusarium spp. with the echinocandin antifungals, and A. terreus with amphotericin B. From the current study, and in the absence of contradictory clinical data suggesting therapeutic benefits, such combinations may be extended to include Acremonium (Sarocladium) and Rasamsonia spp. with amphotericin B, Acremonium (Sarocladium) spp. with itraconazole, Rasamsonia spp., Alternaria spp., and P. variotii with voriconazole, Acremonium spp. with posaconazole, and Acremonium spp., Exophiala spp., P. variotii, and P. lilacinum with caspofungin. Some clinical support exists to suggest that these combinations might not be clinically indicated. For instance, Rasamsonia spp. have been reported as the cause of disseminated infections in CGD patients receiving long-term triazole prophylaxis [51]; several case reports exist of clinical failures with disseminated infections due to Acremonium spp. treated with amphotericin B and itraconazole [52-54]; amphotericin B was ineffective in disseminated human infections caused by P. lilacinum [55], and did not reduce tissue burden in a murine model of disseminated P. lilacinum infection [56]; breakthrough infections and treatment failures have been reported with voriconazole and P. variotii [57,58]. Similarly, for Alternaria spp., we have increasing anecdotal evidence of clinical failures when voriconazole was employed to treat subcutaneous infections (MRL unpublished data).

In the current study, we have also evaluated which of the five test antifungals exert fungicidal effects on common and emerging filamentous fungal pathogens, using a subset of isolates from the NCPF that had been reliably identified using molecular approaches. The amphotericin B, itraconazole, and voriconazole MFC values reported here for Aspergillus spp., E. dermatitidis, S. apiospermum, $R$. arrhizus, and L. corymbifera are in very good agreement with a previous study conducted by us some eight years ago, using different test isolates [31]. Overall, the data confirm the fungicidal activities of amphotericin B, itraconazole, and voriconazole for isolates of Exophiala dermatitidis and for Aspergillus spp. (with the exception of the well-documented resistance to amphotericin B for isolates of A. terreus; Table 6; [38]). Coupled with the lack of demonstrable fungicidal activity of caspofungin against any of the filamentous fungi tested here, these findings also agree extremely well with in vivo neutropenic animal models of invasive aspergillosis [59,60].

\section{Conclusions}

In summary, here we have presented MIC distributions for 4869 clinical isolates of filamentous moulds corresponding to 20 different genera/species. For those organisms that had been previously studied elsewhere, our MIC ranges were in very good agreement with previous reports. The additional MIC ranges presented here for rarer, emerging pathogens (Acremonium spp., Alternaria spp., Exophiala spp., Rasamsonia spp., P. variotii, P. lilacinum, Lo. prolificans) are intended to add to the existing body of literature with the aim of facilitating the development of ECVs for these organisms and the detection of isolates with non-wildtype MICs. However, as ECVs do not predict the clinical outcome of therapy (an isolate with a susceptible or wild-type MIC might not necessarily respond in vivo to that particular antifungal agent), further studies will be required to ascertain the molecular basis for resistance in these particular rarer pathogens in order to establish robust CBPs.

Acknowledgments: We are grateful to Sigma Chemical Co., Janssen Research Foundation, Pfizer UK Ltd, and Merck, Sharp and Dhome for supplying the antifungal agents employed in this study. We are also grateful to the other members of the MRL for their assistance in routine mould susceptibility testing, and to the various laboratories across the UK for submitting their isolates.

Author Contributions: Andrew M. Borman and Elizabeth M. Johnson conceived and designed the experiments; Mark Fraser and Andrew M. Borman performed the cidality experiments; Michael D. Palmer, Adrien Szekely, Zoe Patterson and Marian Houldsworth generated the MIC data for the clinical mould isolates, Andrew M. Borman, Mark Fraser, and Elizabeth M. Johnson analyzed the data; Andrew M. Borman wrote the paper.

Conflicts of Interest: This work was supported in part by a research grant from Gilead Sciences Inc. 


\section{References}

1. Lass-Flörl, C.; Cuenca-Estrella, M. Changes in the epidemiological landscape of invasive mould infections and disease. J. Antimicrob. Chemother. 2017, 72, i5-i11. [CrossRef] [PubMed]

2. Marr, K.A.; Carter, R.A.; Boeckh, M.; Martin, P.; Corey, L. Invasive aspergillosis in allogeneic stem cell transplant recipients: Changes in epidemiology and risk factors. Blood 2002, 100, 4358-4366. [CrossRef] [PubMed]

3. Binder, U.; Lass-Flörl, C. New insights into invasive aspergillosis-From the pathogen to the disease. Curr. Pharm. Des. 2013, 19, 3679-3688. [CrossRef] [PubMed]

4. Kontoyiannis, D.P.; Marr, K.A.; Park, B.J.; Anaissie, E.J.; Walsh, T.J.; Ito, J.; Andes, D.R.; Baddley, J.W.; Brown, J.M.; Brumble, L.M.; et al. Prospective surveillance for invasive fungal infections in hematopoietic stem cell transplant recipients, 2001-2006: Overview of the Transplant-Associated Infection Surveillance Network (TRANSNET) Database. Clin. Infect. Dis. 2010, 50, 1091-1100. [CrossRef] [PubMed]

5. Nir-Paz, R.; Strahilevitz, J.; Shapiro, M.; Keller, N.; Goldschmied-Reouven, A.; Yarden, O.; Block, C.; Polacheck, I. Clinical and epidemiological aspects of infections caused by Fusarium species: A collaborative study from Israel. J. Clin. Microbiol. 2004, 42, 3456-3461. [CrossRef] [PubMed]

6. Garnica, M.; da Cunha, O.M.; Portugal, R.; Maiolino, A.; Colombo, A.L.; Nucci, M.M. Risk factors for invasive fusariosis in patients with acute myeloid leukemia and in hematopoietic cell transplant recipients. Clin. Infect. Dis. 2015, 60, 875-880. [CrossRef] [PubMed]

7. Scheel, C.M.; Hurst, S.F.; Barreiros, G.; Akiti, T.; Nucci, M.M.; Balajee, S.A. Molecular analyses of Fusarium isolates recovered from a cluster of invasive mold infections in a Brazilian hospital. BMC Infect. Dis. 2013, 13, 49. [CrossRef] [PubMed]

8. Cornely, O.A.; Arikan-Akdagli, S.; Dannaoui, E.; Groll, A.H.; Lagrou, K.; Chakrabarti, A.; Lanternier, F.; Pagano, L.; Skiada, A.; Akova, M.; et al. ESCMID and ECMM joint clinical guidelines for the diagnosis and management of mucormycosis 2013. Clin. Microbiol. Infect. 2014, 20, 5-26. [CrossRef] [PubMed]

9. Skiada, A.; Pagano, L.; Groll, A.; Zimmerli, S.; Dupont, B.; Lagrou, K.; Lass-Florl, C.; Bouza, E.; Klimko, N.; Gaustad, P.; et al. European Confederation of Medical Mycology Working Group on Zygomycosis. Zygomycosis in Europe: Analysis of 230 cases accrued by the registry of the European Confederation of Medical Mycology (ECMM) Working Group on Zygomycosis between 2005 and 2007. Clin. Microbiol. Infect. 2011, 17, 1859-1867. [CrossRef] [PubMed]

10. Nucci, M.; Anaissie, E. Fusarium infections in immunocompromised patients. Clin. Microbiol. Rev. 2007, 20, 695-704. [CrossRef] [PubMed]

11. Guarro, J.; Kantarcioglu, A.S.; Horre, R.; Rodriguez-Tudela, J.L.; Estrella, M.C.; Berenguer, J.; de Hoog, G.S. Scedosporium apiospermum: Changing clinical spectrum of a therapy-refractory opportunist. Med. Mycol. 2006, 44, 295-327. [CrossRef] [PubMed]

12. Rodriguez-Tudela, J.L.; Berenguer, J.; Guarro, J.; Kantarcioglu, A.S.; Horre, R.; de Hoog, G.S.; Cuenca-Estrella, M. Epidemiology and outcome of Scedosporium prolificans infection, a review of 162 cases. Med. Mycol. 2009, 47, 359-370. [CrossRef] [PubMed]

13. Chowdhary, A.; Meis, J.F.; Guarro, J.; de Hoog, G.S.; Kathuria, S.; Arendrup, M.C.; Arikan-Akdagli, S.; Akova, M.; Boekhout, T.; Caira, M.; et al. ESCMID/ECMM joint clinical guidelines for the diagnosis and management of systemic phaeohyphomycosis: Diseases caused by black fungi. Clin. Microbiol. Infect. 2014, 20, 47-75. [CrossRef] [PubMed]

14. Anaissie, E.; Kantarjian, H.; Ro, H.; Hopfer, R.; Rolston, R.K.; Fainstein, V.; Bodey, G. The emerging role of Fusarium infections in patients with cancer. Medecine 1988, 67, 77-83. [CrossRef]

15. Berenguer, J.; Rodriguez-Tudela, J.L.; Richard, C.; Alvarez, M.; Sanz, M.A.; Gaztelurrutia, L.; Ayats, J.; Martinez-Suarez, J.V. Deep infections caused by Scedosporium prolificans: A report of 16 cases in Spain and review of the literature. Scedosporium prolificans Spanish study group. Medicine 1997, 76, 256-265. [CrossRef] [PubMed]

16. Cuenca-Estrella, M.; Bassetti, M.; Lass-Florl, C.; Rácil, Z.; Richardson, M.; Rogers, T.R. Detection and investigation of invasive mould disease. J. Antimicrob. Chemother. 2011, 66, i15-i24. [CrossRef] [PubMed]

17. Meis, J.F.; Chowdhary, A.; Rhodes, J.L.; Fisher, M.C.; Verweij, P.E. Clinical implications of globally emerging azole resistance in Aspergillus fumigatus. Philos. Trans. R. Soc. Lond. B. Biol. Sci. 2016, 371, 20150460. [CrossRef] [PubMed] 
18. Ledoux, M.P.; Toussaint, E.; Denis, J.; Herbrecht, R. New pharmacological opportunities for the treatment of invasive mould diseases. J. Antimicrob. Chemother. 2017, 72, i48-i58. [CrossRef] [PubMed]

19. Zhao, Y.; Perez, W.B.; Jiménez-Ortigosa, C.; Hough, G.; Locke, J.B.; Ong, V.; Bartizal, K.; Perlin, D.S. CD101: A novel long-acting echinocandin. Cell. Microbiol. 2016, 18, 1308-1316. [CrossRef] [PubMed]

20. Clinical and Laboratory Standards Institute. Reference Method for Broth Dilution Antifungal Susceptibility Testing of Filamentous Fungi; Approved Standard-Second Edition; Clinical and Laboratory Standards Institute: Wayne, PA, USA, 2008.

21. Subcommittee on Antifungal Susceptibility Testing of the ESCMID European Committee for Antimicrobial Susceptibility Testing. EUCAST technical note on the method for the determination of broth dilution minimum inhibitory concentrations of antifungal agents for conidia-forming moulds. Clin. Microbiol. Infect. 2008, 14, 982-984.

22. Espinel-Ingroff, A.; Chakrabarti, A.; Chowdhary, A.; Cordoba, S.; Dannaoui, E.; Dufresne, P.; Fothergill, A.; Ghannoum, M.; Gonzalez, G.M.; Guarro, J. Multicenter evaluation of MIC distributions for epidemiologic cutoff value definition to detect amphotericin B, posaconazole, and itraconazole resistance among the most clinically relevant species of Mucorales. Antimicrob. Agents Chemother. 2015, 59, 1745-1750. [CrossRef] [PubMed]

23. Espinel-Ingroff, A.; Cuenca-Estrella, M.; Fothergill, A.; Fuller, J.; Ghannoum, M.; Johnson, E.; Pelaez, T.; Pfaller, M.A.; Turnidge, J. Wild-type MIC distributions and epidemiological cutoff values for amphotericin $\mathrm{B}$ and Aspergillus spp. for the CLSI broth microdilution method (M38-A2 document). Antimicrob. Agents Chemother. 2011, 55, 5150-5154. [CrossRef] [PubMed]

24. Espinel-Ingroff, A.; Cuenca-Estrella, M.; Cantón, E. EUCAST and CLSI: Working together towards a harmonized method for antifungal susceptibility testing. Curr. Fungal. Infect. Rep. 2013, 7, 59-67. [CrossRef]

25. Espinel-Ingroff, A.; Fothergill, A.; Fuller, J.; Johnson, E.; Pelaez, T.; Turnidge, J. Wild-type MIC distributions and epidemiological cutoff values for caspofungin and Aspergillus spp. for the CLSI broth microdilution method (M38-A2 document). Antimicrob. Agents Chemother. 2011, 55, 2855-2859. [CrossRef] [PubMed]

26. Espinel-Ingroff, A.; Diekema, D.J.; Fothergill, A.; Johnson, E.; Pelaez, T.; Pfaller, M.A.; Rinaldi, M.G.; Canton, E.; Turnidge, J. Wild-type MIC distributions and epidemiological cutoff values for the triazoles and six Aspergillus spp. for the CLSI broth microdilution method (M38-A2 document). J. Clin. Microbiol. 2010, 48, 3251-3257. [CrossRef] [PubMed]

27. Espinel-Ingroff, A.; Chowdhary, A.; Gonzalez, G.M.; Lass-Flörl, C.; Martin-Mazuelos, E.; Meis, J.; Peláez, T.; Pfaller, M.A.; Turnidge, J. Multicenter study of isavuconazole MIC distributions and epidemiological cutoff values for Aspergillus spp. for the CLSI M38-A2 broth microdilution method. Antimicrob. Agents Chemother. 2013, 57, 3823-3828. [CrossRef] [PubMed]

28. Klepser, M.E.; Wolfe, E.J.; Jones, R.N.; Nightingale, C.H.; Pfaller, M.A. Antifungal pharmacodymanic characteristics of fluconazole and Amphotericin B against Cryptococcus neoformans. Antimicrob. Agents Chemother. 1997, 41, 1392-1401. [PubMed]

29. Douglas, C.M.; Dippolito, J.A.; Shei, G.J.; Meinz, M.; Onishi, J.; Marrinan, J.A.; Li, W.; Abruzzo, G.K.; Flattery, A.; Bartizal, K.; et al. Identification of the FKS1 gene of Candida albicans as the essential target of 1,3- $\beta$-D-glucan synthase inhibitors. Antimicrob. Agents Chemother. 1997, 41, 2471-2479. [PubMed]

30. Manavathu, E.K.; Cutright, J.L.; Chandrasekar, P.H. In vitro susceptibility of itraconazole-resistant isolates of Aspergillus funigatus to voriconazole. Clin. Microbiol. Infect. 1997, 3, 81.

31. Johnson, E.M.; Szekely, A.; Warnock, D.W. In vitro activity of voriconazole, itraconazole and amphotericin B against filamentous fungi. J. Antimicrob. Chemother. 1998, 42, 741-745. [CrossRef] [PubMed]

32. Pfaller, M.A.; Sheehan, D.J.; Rex, J.H. Determination of fungicidal activities against yeasts and moulds: Lessons learned from bacterial testing and the need for standardisation. Clin. Microbiol. Rev. 2004, 17, 268-280.

33. Moore, C.B.; Sayers, N.; Mosquera, J.; Slaven, J.; Denning, D.W. Antifungal drug resistance in Aspergillus. J. Infect. 2000, 41, 203-220. [CrossRef] [PubMed]

34. Rex, J.H.; Pfaller, M.A.; Walsh, T.J.; Chaturvedi, V.; Espinel-Ingroff, A.; Ghannoum, M.A.; Gosey, L.L.; Odds, F.C.; Rinaldi, M.G.; Sheehan, D.J.; et al. Antifungal susceptibility testing: Practical aspects and current challenges. Clin. Microbiol. Rev. 2001, 14, 643-658. [CrossRef] [PubMed] 
35. Salas, V.; Pastor, J.; Calvo, E.; Sutton, D.A.; Fothergill, A.W.; Guarro, J. Evaluation of in vitro activity of voriconazole as predictive of in vivo outcome in a murine Aspergillus fumigatus infection model. Antimicrob. Agents Chemother. 2013, 57, 1404-1408. [CrossRef] [PubMed]

36. Espinel-Ingroff, A. In vitro fungicidal activities of voriconazole, itraconazole and amphotericin B against opportunistic moniliaceous dematiaceous fungi. J. Clin. Microbiol. 2001, 39, 954-958. [CrossRef] [PubMed]

37. Espinel-Ingroff, A.; Fothergill, A.; Peter, J.; Rinaldi, M.G.; Walsh, T.J. Testing conditions for determination of minimum fungicidal concentrations of new and established antifungal agents for Aspergillus spp.: NCCLS collaborative study. J. Clin. Microbiol. 2002, 40, 3204-3208. [CrossRef] [PubMed]

38. Sutton, D.A.; Sanche, S.E.; Revankar, S.G.; Fothergill, A.W.; Rinaldi, M.G. In vitro amphotericin B resistance in clinical isolates of Aspergillus terreus, with a head-to-head comparison to voriconazole. J. Clin. Microbiol. 1999, 37, 2343-2345. [PubMed]

39. Lewis, J.S.; Graybill, J.R. Fungicidal versus fungistatic: What's in a word? Expert Opin. Pharmacother. 2008, 9 , 927-935. [CrossRef] [PubMed]

40. Andes, D. Optimizing antifungal choice and administration. Curr. Med. Res. Opin. 2013, 29, 13-18. [CrossRef] [PubMed]

41. Espinel-Ingroff, A.; Colombo, A.L.; Cordoba, S.; Dufresne, P.J.; Fuller, J.; Ghannoum, M.; Gonzalez, G.M.; Guarro, J.; Kidd, S.E.; Meis, J.F.; et al. International evaluation of MIC distributions and epidemiological cutoff value (ECV) definitions for Fusarium species identified by molecular methods for the CLSI broth microdilution method. Antimicrob. Agents Chemother. 2015, 60, 1079-1084. [CrossRef] [PubMed]

42. Lackner, M.; de Hoog, G.S.; Verweij, P.E.; Najafzadeh, M.J.; Curfs-Breuker, I.; Klaassen, C.H.; Meis, J.F. Species-specific antifungal susceptibility patterns of Scedosporium and Pseudallescheria species. Antimicrob. Agents Chemother. 2012, 56, 2635-2642. [CrossRef] [PubMed]

43. Drogari-Apiranthitou, M.; Mantopoulou, F.D.; Skiada, A.; Kanioura, L.; Grammatikou, M.; Vrioni, G.; Mitroussia-Ziouva, A.; Tsakris, A.; Petrikkos, G. In vitro antifungal susceptibility of filamentous fungi causing rare infections: Synergy testing of amphotericin B, posaconazole and anidulafungin in pairs. J. Antimicrob. Chemother. 2012, 67, 1937-1940. [CrossRef] [PubMed]

44. Houbraken, J.; Giraud, S.; Meijer, M.; Bertout, S.; Frisvad, J.C.; Meis, J.F.; Bouchara, J.P.; Samson, R.A. Taxonomy and antifungal susceptibility of clinically important Rasamsonia species. J. Clin. Microbiol. 2013, 51, 22-30. [CrossRef] [PubMed]

45. Tortorano, A.M.; Richardson, M.; Roilides, E.; van Diepeningen, A.; Caira, M.; Munoz, P.; Johnson, E.; Meletiadis, J.; Pana, Z.D.; Lackner, M.; et al. ESCMID and ECMM joint guidelines on diagnosis and management of hyalohyphomycosis: Fusarium spp., Scedosporium spp. and others. Clin. Microbiol. Infect. 2014, 20, 27-46. [CrossRef] [PubMed]

46. Espinel-Ingroff, A. In vitro antifungal activities of anidulafungin and micafungin, licensed agents and the investigational triazole posaconazole as determined by NCCLS methods for 12,052 fungal isolates: Review of the literature. Rev. Iberoam. Micol. 2003, 20, 121-136. [PubMed]

47. Aguilar-Zapata, D.; Petraitiene, R.; Petraitis, V. Echinocandins: The Expanding Antifungal Armamentarium. Clin. Infect. Dis. 2015, 61, 604-611. [CrossRef] [PubMed]

48. Kim, R.; Khachikian, D.; Reboli, A.C. A comparative evaluation of properties and clinical efficacy of the echinocandins. Expert. Opin. Pharmacother. 2007, 8, 1479-1492. [CrossRef] [PubMed]

49. Rex, J.H.; Pfaller, M.A. Has antifungal susceptibility testing come of age? Clin. Infect. Dis. 2002, 35, 982-989. [CrossRef] [PubMed]

50. Herbrecht, R.; Denning, D.W.; Patterson, T.F.; Bennett, J.E.; Greene, R.E.; Oestmann, J.-W.; Kern, W.V.; Marr, K.A.; Ribaud, P.; Lortholary, O.; et al. Voriconazole versus amphotericin B for primary therapy of invasive aspergillosis. N. Engl. J. Med. 2002, 347, 408-415. [CrossRef] [PubMed]

51. Machouart, M.; Garcia-Hermoso, D.; Rivier, A.; Hassouni, N.; Catherinot, E.; Salmon, A.; Debourgogne, A.; Coignard, H.; Lecuit, M.; Bougnoux, M.-E. Emergence of disseminated infections due to Geosmithia argillacea in patients with chronic granulomatous disease receiving long-term azole antifungal prophylaxis. J. Clin. Microbiol. 2011, 49, 1681-1683. [CrossRef] [PubMed]

52. Herbrecht, R.; Letscher-Bru, V.; Fohrer, C.; Campos, F.; Natarajan-Ame, S.; Zamfir, A.; Waller, J. Acremonium strictum pulmonary infection in a leukemic patient successfully treated with posaconazole after failure of amphotericin B. Eur. J. Clin. Microbiol. Infect. Dis. 2002, 21, 814-817. [CrossRef] [PubMed] 
53. Erbagci, Z.; Tuncel, A.A.; Erkilic, S.; Zer, Y. Successful treatment of antifungal-and cryotherapy-resistant subcutaneous hyalohyphomycosis in an immunocompetent case with topical $5 \%$ imiquimod cream. Mycopathologia 2005, 159, 521-526. [CrossRef] [PubMed]

54. Wang, T.K.; Chiu, W.; Chim, S.; Chan, T.M.; Wong, S.S.; Ho, P.L. Disseminated Ochroconis gallopavum infection in a renal transplant recipient: The first reported case and a review of the literature. Clin. Nephrol. 2003, 60, 415-423. [CrossRef] [PubMed]

55. Van Schooneveld, T.; Freifeld, A.; Lesiak, B.; Kalil, A.; Sutton, D.A.; Iwen, P.C. Paecilomyces lilacinus infection in a liver transplant patient: Case report and review of the literature. Transpl. Infect. Dis. 2008, 10, 117-122. [CrossRef] [PubMed]

56. Rodríguez, M.M.; Pastor, F.J.; Serena, C.; Guarro, J. Posaconazole efficacy in a murine disseminated infection caused by Paecilomyces lilacinus. J. Antimicrob. Chemother. 2009, 63, 361-364. [CrossRef] [PubMed]

57. Chamilos, G.; Kontoyiannis, D.P. Voriconazole-resistant disseminated Paecilomyces variotii infection in a neutropenic patient with leukaemia on voriconazole prophylaxis. J. Infect. 2005, 51, e225-e228. [CrossRef] [PubMed]

58. Feldman, R.; Cockerham, L.; Buchan, B.W.; Lu, Z.; Huang, A.M. Treatment of Paecilomyces variotii pneumonia with posaconazole: Case report and literature review. Mycoses 2016, 59, 746-750. [CrossRef] [PubMed]

59. Petraitiene, R.; Petraitis, V.; Groll, A.H.; Sein, T.; Schaufele, R.L.; Francesconi, A.; Bacher, J.; Avila, N.A.; Walsh, T.J. Antifungal efficacy of caspofungin (MK-0991) in experimental pulmonary aspergillosis in persistently neutropenic rabbits: pharmacokinetics, drug disposition, and relationship to galactomannan antigenemia. Antimicrob. Agents Chemother. 2002, 46, 12-23. [CrossRef] [PubMed]

60. Kirkpatrick, W.R.; Perea, S.; Coc, B.J.; Patterson, T.F. Efficacy of caspofungin alone and in combination with voriconazole in a guinea pig model of invasive aspergillosis. Antimicrob. Agents Chemother. 2002, 46, 2564-2568. [CrossRef] [PubMed]

(C) 2017 by the authors. Licensee MDPI, Basel, Switzerland. This article is an open access article distributed under the terms and conditions of the Creative Commons Attribution (CC BY) license (http://creativecommons.org/licenses/by/4.0/). 